03

\title{
О роли начальных условий в реализации волнового движения в стратифицированной по плотности трехслойной жидкости со свободной поверхностью
}

\author{
(C) С.О. Ширяева, А.И. Григорьев, Л.С. Яковлева
}

Ярославский государственный университет им. П.Г. Демидова, 150000 Ярославль, Россия

e-mail: shir@uniyar.ac.ru

(Поступило в Редакцию 19 января 2016 г. В окончательной редакции 24 мая 2016 г.)

\begin{abstract}
Аналитически исследовано соотношение амплитуд волн, порожденных различными поверхностями раздела сред, в слоисто-неоднородной жидкости. Показано, что амплитуды волн, порожденных второй границей раздела, в зависимости от начальных условий могут быть как больше, так и меньше амплитуд волн, порожденных третьей границей раздела. Отношение амплитуд внешних и внутренних волн между собой сильно зависит от соотношений плотностей жидкости в слоях и их толщин. Влияние начальных условий существенно и проявляется в изменении отношений амплитуд внешних и внутренних волн, а также соотношений амплитуд внутренних волн, порожденных разными поверхностями раздела.
\end{abstract}

DOI: $10.21883 / J T F .2017 .03 .44237 .1739$

Волновое движение на двух поверхностях раздела сред с разной плотностью рассматривалось неоднократно в различных вариантах (см., например, [1-6]). Но теоретический анализ волнового движения на трех поверхностях раздела четырех сред (верхнюю среду моделируем вакуумом) с различными плотностями пока остается экзотическим, хотя тоже не обойден вниманием [7-9]. Роль начальных условий (начальных значений амплитуд, фаз и прочих физических параметров, важных для задачи) в реализации волнового движения в многослойной жидкости также не становилась предметом отдельного исследования.

Следует отметить, что волновое движение на поверхностях раздела обладает целым рядом особенностей, в частности, реализуется нелинейный обмен энергий между гравитационными волнами на одной поверхности при катализационном участии волн, бегущих по другой поверхности [10], а также наблюдается такое явление как эффект „мертвой воды“, реализующийся в многослойной системе несмешивающихся жидкостей $[11,12]$. Можно выделить и другие интересные эффекты [13-16].

Многослойные структуры в естественных условиях встречаются в силу хемоклинной и термоклинной стратификации морской воды. Так, в холодных водах морей и океанов (например, при измерении в Северном Ледовитом океане, проводившемся в точке $85.18^{\circ}$ с.ш. $117.28^{\circ}$ в. д.) можно обнаружить три слоя: $\sim 50 \mathrm{~m}$ воды низкой солености и плотности, „Плавающей“ на поверхности океана, второй слой (более соленой воды) на глубине $\sim 150 \mathrm{~m}$, при этом растет и плотность воды, третий слой это глубокий (более $\sim 150 \mathrm{~m}$ ) с почти постоянной соленостью и температурой (ссылку на эту работу легко найти в Интернете). Таким образом, возникает многослойная структура с несколькими границами раздела, и исследование волнового движения в ней представляется актуальным, например, в связи с проблемами судоходства. Исследованию роли начальных условий при реализации волнового движения в многослойных системах (с постоянной внутри слоев плотностью) на основе решения модельной задачи и посвящена настоящая работа.

\section{Формулировка задачи}

Рассмотрим идеализированную модельную систему, состоящую из бесконечно глубокого слоя жидкости плотностью $\rho_{3}$, на котором находятся два жидких слоя толщиной $h_{2}$, плотностью $\rho_{2}$ и толщиной $h_{1}$, плотностью $\rho_{1}$ соответственно. Над свободной границей раздела находится воздух с давлением $P_{0}$ (в нижеследующем изложении будем моделировать эту среду вакуумом). Все жидкости предполагаются идеальными, несжимаемыми, несмешиваемыми. Система находится в поле сил тяжести g. Введем декартову систему координат, орт $\mathbf{n}_{z}$ которой направлен вертикально вверх $\left(\mathbf{n}_{z} \|-\mathbf{g}\right)$, а плоскость $z=0$ совпадает с гранией раздела нижнего и среднего слоев. Орт $\mathbf{n}_{x}$ вводится параллельно невозмущенной волновым движением границе раздела жидкостей так, чтобы начало системы координат находилось на границе раздела нижней жидкости с массовой плотностью $\rho_{3}$ и лежащей на ней жидкости с плотностью $\rho_{2}$. При таком введении декартовых координат средний слой занимает пространство $0 \leq z \leq h_{2}$, верхний $h_{2} \leq z \leq h_{2}+h_{1}$, а нижний $-z \leq 0$.

При волновом возмущении границ раздела сред свободная поверхность верхней жидкости описывается уравнением $F_{1}(x, z, t) \equiv z-\xi_{1}(x, t)-\left(h_{1}+h_{2}\right)=0$, где $\xi_{1}(x, t)$ - малое отклонение поверхности верхней жидкости от невозмущенного уровня $z=h_{1}+h_{2}$; уравнение границы раздела верхней и средней жидкостей 
будет иметь вид $F_{2}(x, z, t) \equiv z-\xi_{2}(x, t)-h_{2}=0$, где $\xi_{2}(x, t)$ - малое отклонение границы раздела жидкостей от невозмущенного уровня $z=h_{2}$, а граница раздела средней и нижней жидкостей описывается уравнением $F_{3}(x, z, t) \equiv z-\xi_{3}(x, t)=0$, где $\xi_{3}(x, t)$ - малое отклонение границы раздела жидкостей от невозмущенного уровня $z=0$.

Зададимся целью рассмотрения особенности волновых движений в описанной системе на основе анализа дисперсионного соотношения, ограничиваясь рассмотрением гравитационных волн, т.е. волн, длина которых много больше капиллярной постоянной жидкости. Задачу будем решать в линейном приближении по безразмерным амплитудам волновых возмущений поверхностей раздела сред.

Математическая формулировка задачи состоит из уравнений непрерывности и уравнений Эйлера для трех сред в соответствующих областях пространства:

$$
\begin{gathered}
\operatorname{div} \mathbf{V}_{j}(\mathbf{r}, t)=0, \quad j=(1,2,3), \\
\frac{\partial \mathbf{V}_{j}(\mathbf{r}, t)}{\partial t}+\left(\mathbf{V}_{j}(\mathbf{r}, t) \nabla\right) \mathbf{V}_{j}(\mathbf{r}, t)=-\frac{1}{\rho_{j}} \nabla P_{j}(\mathbf{r}, t)+\mathbf{g} .
\end{gathered}
$$

Здесь $\mathbf{V}_{j}(\mathbf{r}, t)$ - поля скоростей течения жидкостей, $\rho_{j}-$ плотности жидкостей, $P_{j}(\mathbf{r}, t)-$ гидродинамические давления в слоях.

Граничные условия определяют характеристики движения жидкости на ограничивающих поверхностях:

$$
\begin{gathered}
z \rightarrow-\infty: \quad \mathbf{V}_{3}(\mathbf{r}, t) \rightarrow 0, \\
F_{1}=0 \Leftrightarrow z=\left(h_{1}+h_{2}\right)+\xi_{1}(x, t): \\
\frac{d F_{1}}{d t}=\left(\frac{\partial F_{1}}{\partial t}+\mathbf{V}_{j}(\mathbf{r}, t) \nabla F_{1}\right)=0, \\
P_{1}(\mathbf{r}, t)=P_{0}, \\
F_{2}=0 \Leftrightarrow z=h_{2}+\xi_{2}(x, t): \\
\frac{d F_{2}}{d t}=\left(\frac{\partial F_{2}}{\partial t}+\mathbf{V}_{1}(\mathbf{r}, t) \nabla F_{2}\right)=0, \\
\frac{d F_{2}}{d t}=\left(\frac{\partial F_{2}}{\partial t}+\mathbf{V}_{2}(\mathbf{r}, t) \nabla F_{2}\right)=0, \\
P_{2}(\mathbf{r}, t)=P_{1}(\mathbf{r}, t), \\
F_{3}=0 \Leftrightarrow z=\xi_{3}(x, t): \\
\frac{d F_{3}}{d t}=\left(\frac{\partial F_{3}}{\partial t}+\mathbf{V}_{2}(\mathbf{r}, t) \nabla F_{3}\right)=0, \\
\frac{d F_{3}}{d t}=\left(\frac{\partial F_{3}}{\partial t}+\mathbf{V}_{3}(\mathbf{r}, t) \nabla F_{3}\right)=0, \\
P_{2}(\mathbf{r}, t)=P_{3}(\mathbf{r}, t) .
\end{gathered}
$$

Выписанная система уравнений и граничных условий представляет собой полную математическую формулировку задачи.

\section{Вывод дисперсионного уравнения}

Процедура решения задачи эквивалентна ранее использованной [9]. Поэтому отметим лишь, что искать решения уравнений Лапласа для гидродинамических потенциалов $\Psi_{j}(\mathbf{r}, t)$ (принимаем, что $\mathbf{V}_{j}(\mathbf{r}, t) \equiv \Delta \Psi_{j}(\mathbf{r}, t)$, где $j=1,2,3$ ) будем в виде

$$
\begin{gathered}
\Psi_{1}(x, z, t)=\left[D_{1}(t) \exp (k z)+D_{2}(t) \exp (-k z)\right] \exp (i k x), \\
\Psi_{2}(x, z, t)=\left[D_{3}(t) \exp (k z)+D_{4}(t) \exp (-k z)\right] \exp (i k x), \\
\Psi_{3}(x, z, t)=D_{5}(t) \exp (k z) \exp (i k x),
\end{gathered}
$$

а выражения для возмущений $\xi_{j}(x, t)$ как

$$
\begin{gathered}
\xi_{1}(x, t)=A_{1}(t) \exp (i k x), \quad \xi_{2}(x, t)=A_{2}(t) \exp (i k x), \\
\xi_{3}(x, t)=A_{3}(t) \exp (i k x) .
\end{gathered}
$$

Если принять

$$
A_{j}(t)=\alpha_{j} \exp (i \omega t)
$$

то несложно получить (по аналогии с [9]) дисперсионное уравнение задачи в виде бикубического относительно частоты волн $\omega$ алгебраического уравнения

$$
\begin{gathered}
\left(\omega^{2}-g k\right)\left(a \omega^{4}+b \omega^{2}+c\right)=0, \\
a=\left\{\rho _ { 2 } \left[\rho_{1} \operatorname{ch}\left(h_{2} k\right) \operatorname{sh}\left(h_{1} k\right)+\rho_{2} \operatorname{ch}\left(h_{1} k\right) \operatorname{sh}\left(h_{2} k\right)\right.\right. \\
\left.+\rho_{3}\left[\rho_{1} \operatorname{sh}\left(h_{1} k\right) \operatorname{sh}\left(h_{2} k\right)+\rho_{2} \operatorname{ch}\left(h_{1} k\right) \operatorname{ch}\left(h_{2} k\right)\right]\right\}, \\
b=g k \rho_{2}\left\{\rho_{1} \operatorname{sh}\left(h_{1} k\right)\left[\operatorname{ch}\left(h_{2} k\right)+\operatorname{sh}\left(h_{2} k\right)\right]\right. \\
\left.+\rho_{2}\left(\operatorname{ch}\left(h_{1} k\right)-\operatorname{sh}\left(h_{1} k\right)\right) \operatorname{sh}\left(h_{2} k\right)-\rho_{3} \operatorname{sh}\left(\left(h_{1}+h_{2}\right) k\right)\right\}, \\
c=g^{2} k^{2}\left(\rho_{1}-\rho_{2}\right)\left(\rho_{2}-\rho_{3}\right) \operatorname{sh}\left(h_{1} k\right) \operatorname{sh}\left(h_{2} k\right),
\end{gathered}
$$

где $k$ имеет смысл волнового числа, а $\alpha_{j}-$ неизвестные амплитуды волн на поверхностях раздела.

Решения уравнения (1) элементарно выписываются в аналитическом виде

$$
\begin{gathered}
\omega_{1}^{2}=g k, \quad \omega_{2}^{2}=\frac{1}{2 a}\left(-b-\sqrt{b^{2}-4 a c}\right), \\
\omega_{3}^{2}=\frac{1}{2 a}\left(-b+\sqrt{b^{2}-4 a c}\right) .
\end{gathered}
$$

Решение $\omega_{1}^{2}$ соответствует свободной поверхности верхнего слоя, $\omega_{2}^{2}-$ границе раздела двух слоев конечной толщины: верхнего и среднего, а решение $\omega_{3}^{2}-$ границе раздела среднего слоя конечной толщины и бесконечно глубокой нижней жидкости. Это следует из поведения выписанных корней при рассмотрении предельного перехода к двуслойной системе. Такой предельный переход можно осуществить, устремляя плотность среднего слоя к плотности либо верхней жидкости $\rho_{2} \rightarrow \rho_{1}$, либо нижней $\rho_{2} \rightarrow \rho_{3}$. В обоих случаях исчезает граница раздела двух слоев конечной толщины, и при этом корень дисперсионного уравнения $\omega_{2}^{2}$ обращается в нуль. В то же время решение $\omega_{3}^{2}$ принимает вид, совпадающий с решением для внутренних волн в двуслойной задаче, только в первом случае толщина слоя равна $h_{1}+h_{2}$, а во втором $-h_{1}$. 


\section{Отношения амплитуд внутренних и внешних волн и выражения для форм поверхностей раздела сред}

Для каждого из корней дисперсионного уравнения несложно найти отношения амплитуд волн, распространяющихся по разным поверхностям раздела:

$$
\begin{aligned}
& \eta_{21} \equiv \frac{\alpha_{2}}{\alpha_{1}}=\frac{1}{\omega_{j}^{2}}\left[\omega_{j}^{2} \operatorname{ch}\left(h_{1} k\right)-g k \operatorname{sh}\left(h_{1} k\right)\right], \\
\eta_{31} \equiv & \frac{\alpha_{3}}{\alpha_{1}}= \\
= & \frac{\rho_{2}\left(\omega_{j}^{2} \operatorname{ch}\left(h_{1} k\right)-g k \operatorname{sh}\left(h_{1} k\right)\right)}{\omega_{j}^{2}\left[\rho_{2} \operatorname{ch}\left(h_{2} k\right)+\rho_{3} \operatorname{sh}\left(h_{2} k\right)\right]-g k\left(\rho_{3}-\rho_{2}\right) \operatorname{sh}\left(h_{2} k\right)}, \\
\eta_{32} \equiv & \frac{\alpha_{3}}{\alpha_{2}}= \\
= & \frac{\rho_{2} \omega_{j}^{2}}{\omega_{j}^{2}\left[\rho_{2} \operatorname{ch}\left(h_{2} k\right)+\rho_{3} \operatorname{sh}\left(h_{2} k\right)\right]-g k\left(\rho_{3}-\rho_{2}\right) \operatorname{sh}\left(h_{2} k\right)} .
\end{aligned}
$$

Вводя обозначения $\alpha_{n j} \equiv \alpha_{n}\left(\omega_{j}^{2}\right)$, где $n, j=1,2,3$, запишем общие решения для функций $\xi_{n}(x, t)$, описывающих формы свободной поверхности и границ раздела слоев, в виде

$$
\begin{aligned}
& \xi_{1}(x, t)=\alpha_{11} \exp \left[i\left(k x-\omega_{1} t\right)\right]+\alpha_{12} \exp \left[i\left(k x-\omega_{2} t\right)\right] \\
&+\alpha_{13} \exp \left[i\left(k x-\omega_{3} t\right)\right], \\
& \xi_{2}(x, t)= \eta_{21}\left(\omega_{1}^{2}\right) \alpha_{11} \exp \left[i\left(k x-\omega_{1} t\right)\right] \\
&+\eta_{21}\left(\omega_{2}^{2}\right) \alpha_{12} \exp \left[i\left(k x-\omega_{2} t\right)\right] \\
&+\eta_{21}\left(\omega_{3}^{2}\right) \alpha_{13} \exp \left[i\left(k x-\omega_{3} t\right)\right], \\
& \xi_{3}(x, t)= \eta_{31}\left(\omega_{1}^{2}\right) \alpha_{11} \exp \left[i\left(k x-\omega_{1} t\right)\right] \\
&+\eta_{31}\left(\omega_{2}^{2}\right) \alpha_{12} \exp \left[i\left(k x-\omega_{2} t\right)\right] \\
&+\eta_{31}\left(\omega_{3}^{2}\right) \alpha_{13} \exp \left[i\left(k x-\omega_{3} t\right)\right] .
\end{aligned}
$$

Представляя в общем случае комплексные амплитуды $\alpha_{11}, \alpha_{12}, \alpha_{13}$ в экспоненциальной форме $\alpha_{1 j}=a_{j} \exp \left(i b_{j}\right)$, найдем значения вещественных амплитуд $a_{j}$ и фаз $b_{j}$ из следующих начальных условий:

$$
\begin{gathered}
t=0: \quad \xi_{n}(x)=\xi_{n} \exp (i k x), \\
\frac{\partial \xi_{n}(x)}{\partial t}=0, \quad n=1,2,3 .
\end{gathered}
$$

Каждая из фаз $b_{j}$ может принимать одно из двух значений: либо нуль, либо $\pi$. При этом выражения для соответствующей амплитуды $a_{j}$ отличаются только знаком. Поскольку в дальнейшем исследовании интерес представляет сравнение абсолютных величин амплитуд волн, то для примера все фазы $b_{j}$ можно принять равными нулю, а амплитуды $a_{j}$, а следовательно, и комплексные амплитуды $\alpha_{1 j}$ будут определяться выражениями

$$
\begin{aligned}
& \xi_{1}\left(\eta_{21}\left(\omega_{2}^{2}\right) \eta_{31}\left(\omega_{2}^{2}\right)-\eta_{21}\left(\omega_{3}^{2}\right) \eta_{31}\left(\omega_{2}^{2}\right)\right)+ \\
& \alpha_{11}=\frac{+\xi_{2}\left(\eta_{31}\left(\omega_{2}^{2}\right)-\eta_{31}\left(\omega_{3}^{2}\right)\right)-\xi_{3}\left(\eta_{21}\left(\omega_{2}^{2}\right)-\eta_{21}\left(\omega_{3}^{2}\right)\right)}{\eta_{21}\left(\omega_{1}^{2}\right)\left(\eta_{31}\left(\omega_{2}^{2}\right)-\eta_{31}\left(\omega_{3}^{2}\right)\right)-}, \\
& -\eta_{21}\left(\omega_{2}^{2}\right)\left(\eta_{31}\left(\omega_{1}^{2}\right)-\eta_{31}\left(\omega_{3}^{2}\right)\right)+\eta_{21}\left(\omega_{3}^{2}\right)\left(\eta_{31}\left(\omega_{1}^{2}\right)-\eta_{31}\left(\omega_{2}^{2}\right)\right) \\
& \alpha_{12}=\frac{\xi_{1}\left(\eta_{21}\left(\omega_{1}^{2}\right) \eta_{31}\left(\omega_{3}^{2}\right)-\eta_{21}\left(\omega_{3}^{2}\right) \eta_{31}\left(\omega_{1}^{2}\right)\right)+}{+\xi_{2}\left(\eta_{31}\left(\omega_{1}^{2}\right)-\eta_{31}\left(\omega_{3}^{2}\right)\right)-\xi_{3}\left(\eta_{21}\left(\omega_{1}^{2}\right)-\eta_{21}\left(\omega_{3}^{2}\right)\right)}, \\
& -\eta_{21}\left(\omega_{2}^{2}\right)\left(\eta_{31}\left(\omega_{1}^{2}\right)-\eta_{31}\left(\omega_{3}^{2}\right)\right)+\eta_{21}\left(\omega_{3}^{2}\right)\left(\eta_{31}\left(\omega_{1}^{2}\right)-\eta_{31}\left(\omega_{2}^{2}\right)\right) \\
& \xi_{1}\left(\eta_{21}\left(\omega_{2}^{2}\right) \eta_{31}\left(\omega_{1}^{2}\right)-\eta_{21}\left(\omega_{1}^{2}\right) \eta_{31}\left(\omega_{2}^{2}\right)\right)- \\
& \alpha_{13}=\frac{-\xi_{2}\left(\eta_{31}\left(\omega_{1}^{2}\right)-\eta_{31}\left(\omega_{2}^{2}\right)\right)+\xi_{3}\left(\eta_{21}\left(\omega_{1}^{2}\right)-\eta_{21}\left(\omega_{2}^{2}\right)\right)}{\eta_{21}\left(\omega_{1}^{2}\right)\left(\eta_{31}\left(\omega_{2}^{2}\right)-\eta_{31}\left(\omega_{3}^{2}\right)\right)-} . \\
& -\eta_{21}\left(\omega_{2}^{2}\right)\left(\eta_{31}\left(\omega_{1}^{2}\right)-\eta_{31}\left(\omega_{3}^{2}\right)\right)+\eta_{21}\left(\omega_{3}^{2}\right)\left(\eta_{31}\left(\omega_{1}^{2}\right)-\eta_{31}\left(\omega_{2}^{2}\right)\right)
\end{aligned}
$$

\section{Зависимости отношений амплитуд внутренних волн к амплитуде внешних волн от физических параметров системы}

На рис. 1 представлены зависимости отношений амплитуд волн от толщины верхнего слоя $h_{1}$ для корня дисперсионного уравнения $\omega_{3}$. Из полученного рисунка следует, что изменение толщины верхнего слоя оказывает заметное влияние на отношение амплитуд. Отметим, что, как показывают расчеты для корня $\omega_{2}$, амплитуда $a_{2}$ больше амплитуды $a_{3}$, и обе примерно линейно увеличиваются с ростом $h_{1}$, а в случае корня $\omega_{3}$, наоборот: $a_{3}$ превышает $a_{2}$ (рис. 1). Таким образом, наблюдается аналог эффекта „мертвой воды“ для внутренних волн: амплитуда волн, порожденных более „глубокой“ третьей границей раздела, больше амплитуды волн на „внешней“ второй границе.

C ростом $h_{1}$ отношение $\left|\eta_{21}\right|$ снизу, а отношение $\left|\eta_{31}\right|$ сверху стремятся к единой наклонной растущей асимптотике. Отношение $\left|\eta_{32}\right|$ с ростом $h_{1}$ слабо убывает, стремясь к единице. Увеличение $\left|\eta_{21}\right|$ и $\left|\eta_{31}\right|$, по-видимому, вызвано тем, что с ростом толщины верхнего слоя $h_{1}$ влияние волн, порожденных внутренними границами раздела, на свободную поверхность уменьшается и, как следствие, уменьшается амплитуда $a_{1}$, приводя к росту отношений $\left|\eta_{21}\right|$ и $\left|\eta_{31}\right|$.

На рис. 2 представлены аналогичные зависимости отношений амплитуд от толщины среднего слоя $h_{2}$, для корней $\omega_{2}$ и $\omega_{3}$. В случае корня $\omega_{2}$ амплитуда волн на второй границе превышает амплитуду волн на третьей границе, а для $\omega_{3}$ вследствие эффекта „мертвой воды“ - наоборот. Из рис. 2, $a$ видно, что увеличение толщины среднего слоя уменьшает значения отношения амплитуд волн, в то время как изменение толщины верхнего слоя $h_{1}$ их увеличивало. Для корня $\omega_{3}$ изменение $h_{2}$ сказывается на значениях отношений амплитуд противоположным образом: с ростом $h_{2}$ отношение $\left|\eta_{21}\right|$ 


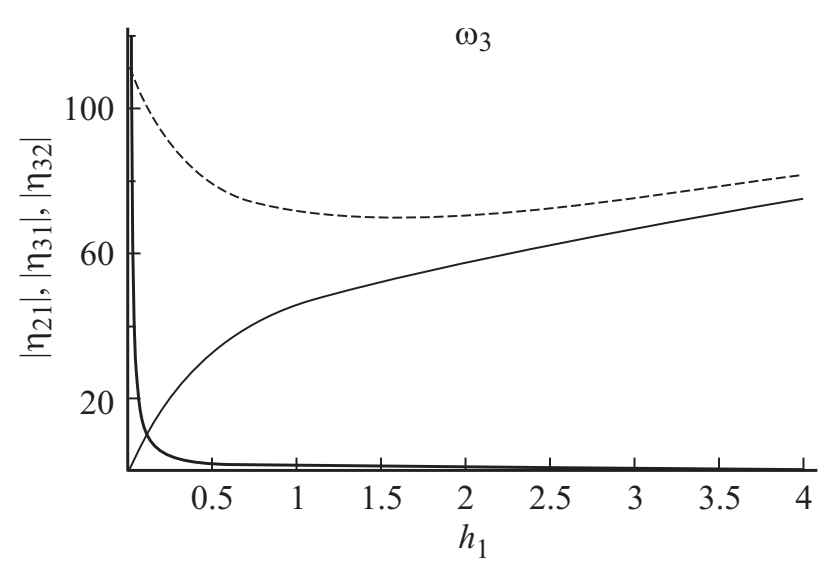

Рис. 1. Зависимости отношения амплитуд от толщины верхнего слоя $h_{1}$ для $\omega_{3}: g=1, k=0.1, \rho_{1}=1, \rho_{2}=1.01, \rho_{3}=1.02$, $h_{2}=1$. На этом рисунке и на всех последующих: $\left|\eta_{21}\right|$ - тонкие сплошные линии, $\left|\eta_{31}\right|$ - штриховые линии, $\left|\eta_{32}\right|$ - толстые сплошные линии.
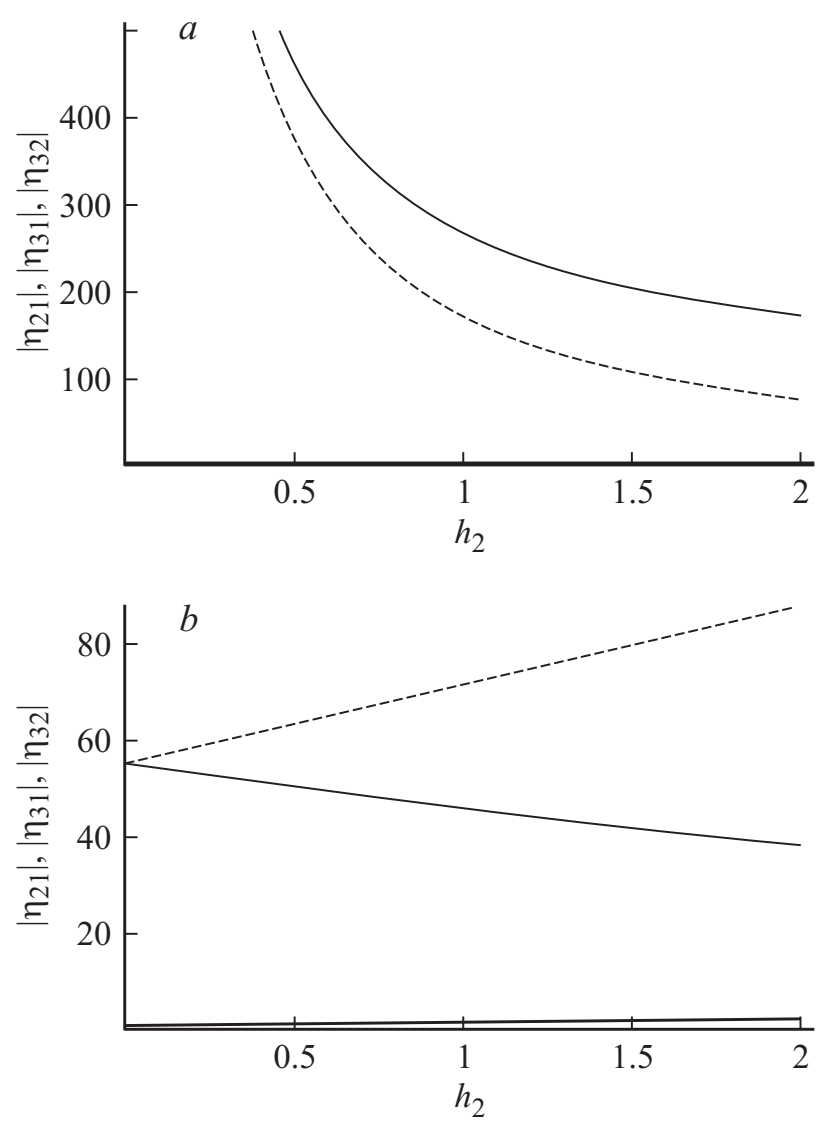

Рис. 2. Зависимости отношения амплитуд от толщины среднего слоя $h_{2}$. Рассчитано при: $g=1, k=0.1, \rho_{1}=1, \rho_{2}=1.01$, $\rho_{3}=1.02, h_{1}=1 . a-\omega_{2}, b-\omega_{3}$. На рис. 2, $a$ кривая, соответствующая $\eta_{32}$, сливается с осью абсцисс.

уменьшается, а $\left|\eta_{31}\right|$ растет (рис. $\left.2, b\right)$. Объяснить такой вид зависимостей, как и в случае, представленном на рис. 1, можно уменьшающимся с ростом $h_{2}$ влиянием волн, порожденных самой нижней границей раздела, на возбуждение волн, как на средней, так и на верхней поверхностях. Непропорциональное уменьшение амплитуд $a_{1}$ и $a_{2}$, по всей видимости, и является причиной описанного неодинакового поведения отношений $\left|\eta_{21}\right|$ и $\left|\eta_{31}\right|$. Отметим, что, как и в предыдущем случае (рис. 1), значения относительных амплитуд для корня $\omega_{2}$ существенно выше, чем для корня $\omega_{3}$. Отношение $\left|\eta_{32}\right|$ в обоих случаях с ростом $h_{2}$ слабо растет.

На рис. 3 изображены зависимости отношения амплитуд от плотности среднего слоя $\rho_{2}$ при $\rho_{1}=1$. На рис. $3, a$ (для корня $\omega_{2}$ ) отношение $\left|\eta_{21}\right|$ стремительно растет при приближении плотности среднего слоя как к плотности верхнего слоя $\rho_{1}=1$, так и к плотности нижнего $\rho_{3}$. Аналогично быстрый рост наблюдается и для отношений $\left|\rho_{31}\right|$ при уменьшении разности плотностей среднего и нижнего слоев. Как уже упоминалось выше, стремление плотности $\rho_{2}$ как к $\rho_{1}$, так и к $\rho_{3}$ соответствует переходу к двуслойной системе, когда средняя граница раздела слоев конечной толщины исчезает, а частота $\omega_{2}$ обращается в нуль. Очевидно, что рассмотрение волн, порожденных этой границей, в этом случае теряет смысл. Другими словами, следует понимать, что область применимости полученного решения для трехслойной системы по плотности $\rho_{2}$ имеет свои пределы. Сместив границы этой области от значений $\rho_{1}$ и $\rho_{3}$
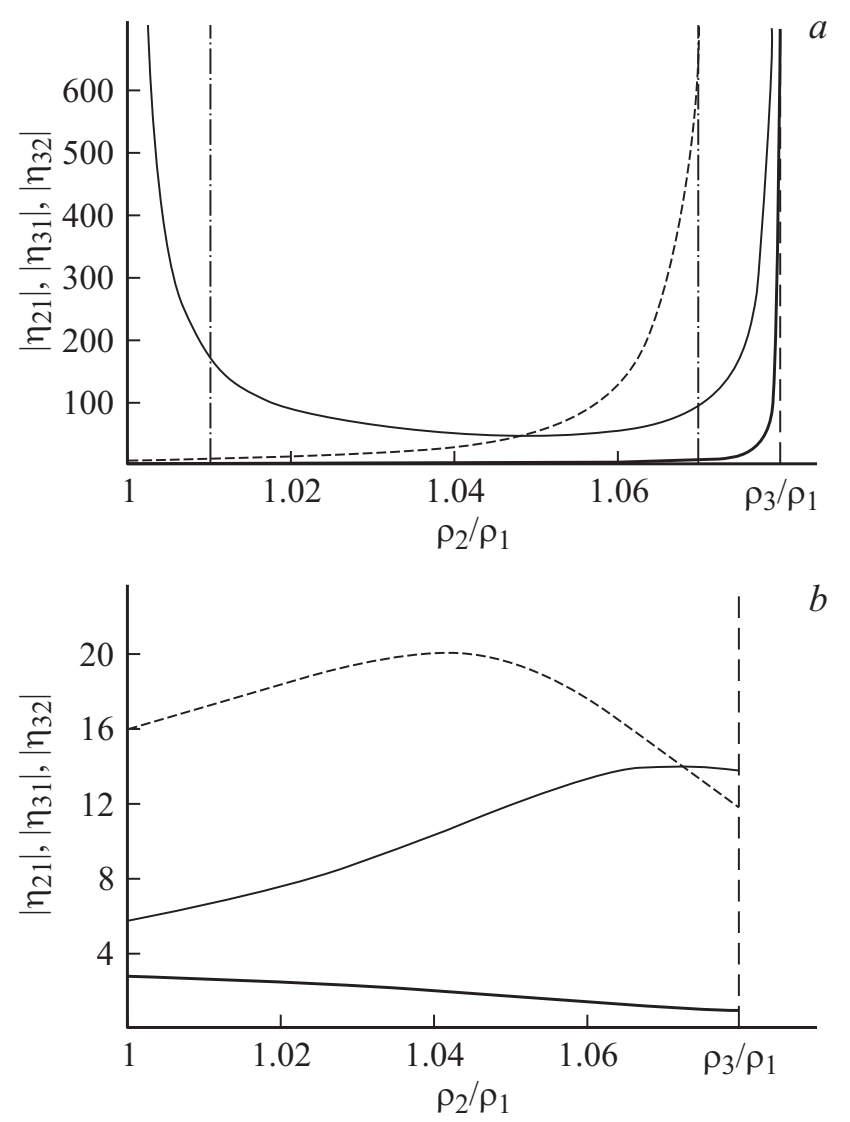

Рис. 3. Зависимости отношения амплитуд от плотности верхнего слоя $\rho_{2}$. Рассчитано при: $g=1, k=0.1, \rho_{1}=1, \rho_{3}=1.08$, $h_{1}=1, h_{2}=1.5 . a-\omega_{2}, b-\omega_{3}$. 
(штрихпунктирные линии на рис. 3,a), получим, что в выделенной области никакого безграничного нарастания амплитуд не происходит, и их отношения ведут себя вполне адекватно.

Для корня $\omega_{3}$ из рис. $3, b$ видно, что отношение амплитуд $\left|\eta_{21}\right|$ плавно нарастает при изменении плотности $\rho_{2}$ в диапазоне от $\rho_{1}$ до $\rho_{3}$, а отношение $\left|\eta_{31}\right|$ ведет себя немонотонно. Однако в отличие от предыдущего случая оба они остаются конечными. Это объясняется тем, что корень $\omega_{3}$ при $\rho_{2} \rightarrow \rho_{1}$ и $\rho_{2} \rightarrow \rho_{3}$ не исчезает, а принимает вид, совпадающий с решением для частоты внутренних волн в двуслойной задаче. При $\rho_{2} \rightarrow \rho_{1}$ толщина слоя равна $h_{1}+h_{2}$, а при $\rho_{2} \rightarrow \rho_{3}$ соответственно $h_{1}$. Заметим, что и в этом случае область правомерного рассмотрения отношений $\left|\eta_{21}\right|$ и $\left|\eta_{32}\right|$ по плотности $\rho_{2}$ уже диапазона $\left[\rho_{1}, \rho_{3}\right]$, поскольку при $\rho_{2}=\rho_{1}$ и $\rho_{2}=\rho_{3}$ система превращается в двуслойную и рассмотрение амплитуды $\alpha_{2}$, а следовательно, и отношений $\left|\eta_{21}\right|$ и $\left|\eta_{32}\right|$ теряет смысл.

Характерно, что амплитуды внутренних волн как $a_{2}$, так и $a_{3}$ в данном случае (рис. $3, b$ ) не столь значительно в сравнении с рис. $2, b$ превосходят амплитуду волн $a_{1}$ на свободной поверхности. Это связано с бо́льшим, чем на рис. $2, b$, значением плотности $\rho_{3}$, принятом при расчетах. Отношение $\left|\eta_{32}\right|$ на рис. $3, b$ при приближении плотности среднего слоя $\rho_{2}$ к плотности нижнего $\rho_{3}$ плавно уменьшается.

На рис. 4 изображены аналогичные зависимости отношений амплитуд от плотности нижнего слоя $\rho_{3}$ при $\rho_{1}=1$. Для обоих режимов внутренних волн (т.е. для корней $\omega_{2}$ и $\omega_{3}$ ) отношения $\left|\eta_{21}\right|$ и $\left|\eta_{31}\right|$ существенно возрастают при уменьшении разности плотностей $\rho_{3}$ и $\rho_{2}$ (т. е. при $\left.\rho_{3} \rightarrow \rho_{2}\right)$. При увеличении $\rho_{3}$ амплитуды внутренних волн либо медленно убывают (рис. $4, b$ ), либо выходят на горизонтальную асимптотику (рис. 4, $a$ ). Видно, что амплитуда волн на средней границе раздела сред для корня $\omega_{2}$ превышает амплитуду внутренних волн на нижней границе раздела сред, а для корня $\omega_{3}$ наоборот.

На рис. 4 штрихпунктирной линией условно изображена граница области применимости трехслойной модели, которая при $\rho_{3} \rightarrow \rho_{2}$ переходит в двухслойную систему. Из сравнения рис. $4, a$ и $4, b$ видно, что значения амплитуд для корня $\omega_{2}$ на порядок больше, чем для $\omega_{3}$. Отношение $\eta_{32}$ с ростом $\rho_{3}$ для корня $\omega_{2}$ уменьшается, а для корня $\omega_{3}$ увеличивается.

Следует отметить, что приведенные рисунки демонстрируют лишь качественные тенденции, поскольку получены из модельных расчетов. Для точного численного соответствия в каждом конкретном случае необходимо использовать при расчетах конкретные значения плотностей и толщин слоев, которые сильно различаются для разных проливов и разных океанов.

Из приведенных рисунков видно, что влияние начальных условий (в общем случае под ними понимается кроме определения начальных амплитуд и фаз внешних и внутренних волн задание всей совокупности важных для
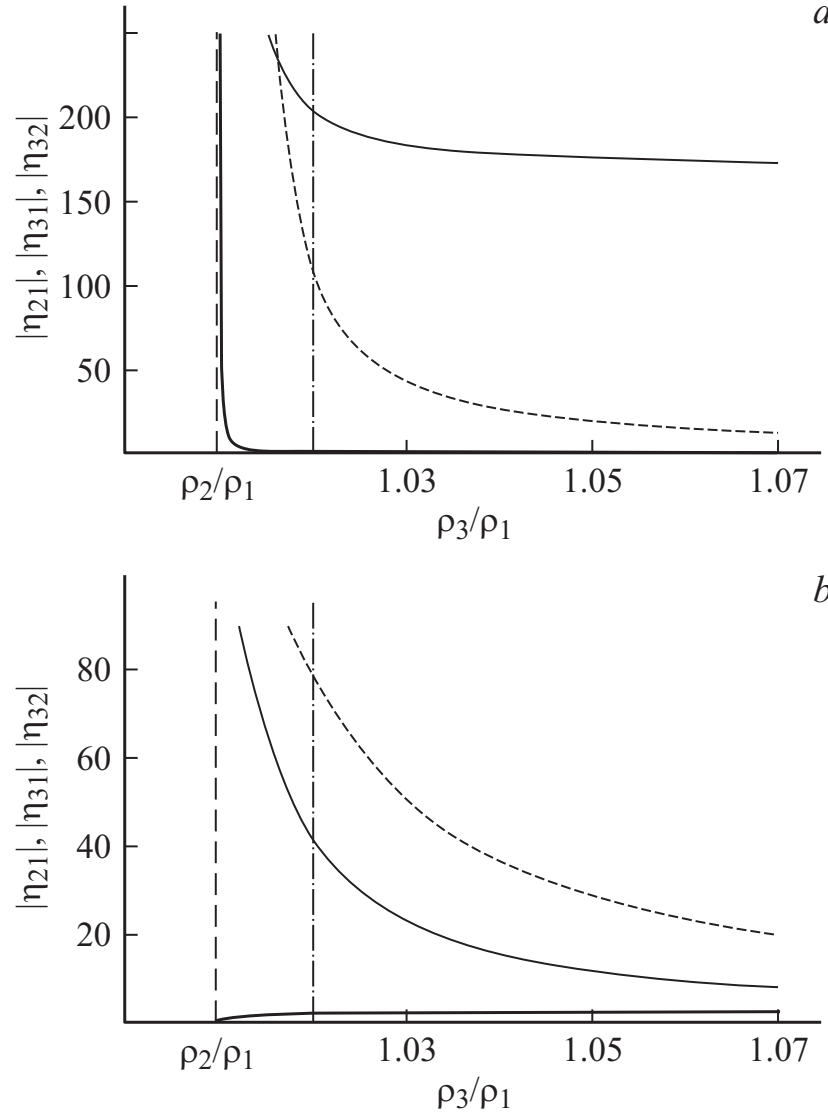

Рис. 4. Зависимости отношения амплитуд от плотности нижнего слоя $\rho_{3}$. Рассчитано при: $g=1, k=0.1, \rho_{1}=1, \rho_{2}=1.01$, $h_{1}=1, h_{2}=1.5 . a-\omega_{2}, b-\omega_{3}$.

реализации волнового движения физических условий) на физическую картину реализующихся на поверхностях раздела сред волновых движений жидкости весьма существенно.

При начальном возмущении верхней границы амплитуды волн на внутренних поверхностях раздела меньше амплитуды волны на свободной поверхности и составляют десятые доли от значения последней, что характерно для режима колебаний „сплошной среды“. Расчеты показывают, что с течением времени происходит сдвиг фаз между внутренними волнами и волнами на свободной поверхности.

При начальном возмущении средней границы раздела амплитуды волн на среднем слое жидкости превышают амплитуды волн как на свободной поверхности, так и нижнем слое. Причем амплитуды поверхностных волн на один-два порядка ниже амплитуд внутренних волн, что является характерной особенностью режима колебаний „внутренних волн“. С течением времени образуется сдвиг фаз между волнами на разных поверхностях раздела.

При начальном возмущении нижней границы раздела наибольшей амплитудой обладают волны именно на этой поверхности, амплитуды волн на средней поверх- 
ности сравнимы с нижней, а амплитуды поверхностных волн существенно меньше. Таким образом, рассматриваемый случай также можно отнести к режиму „внутренних волн".

Все проведенные расчеты выполнены для случая, когда значения плотностей всех трех слоев сравнимы друг с другом $\rho_{3} \sim \rho_{2} \sim \rho_{1}$. Анализ показал, что в такой ситуации при возбуждении верхней границы раздела реализуется режим колебаний „сплошной среды“, а при возбуждении как средней, так и нижней - режимы „внутренних волн“.

Рассмотрим случай, когда сравнимые между собой плотности нижнего и среднего слоев существенно превосходят плотность верхнего слоя $\rho_{3} \sim \rho_{2}>\rho_{1}$. В такой ситуации режим внутренних волн реализуется лишь при начальном возмущении нижнего слоя, т.е. поверхности раздела двух сред, плотности которых сравнимы между собой. При начальном возмущении как свободной поверхности, так и поверхности среднего слоя амплитуды волн на всех трех границах раздела сравнимы между собой. Следовательно, эти режимы колебаний можно условно отнести к режиму „сплошной среды“. Таким образом, режим движения „внутренних волн“ тем ярче проявляется, чем меньше разность плотностей сред, граница раздела которых возбуждается.

Однако возможность реализации того либо иного режимов движения зависит также и от соотношения между длиной волны и толщиной слоев. При начальном возмущении границ раздела верхнего и среднего слоев, плотности которых сушественно различны $\rho_{3} \sim \rho_{2}>\rho_{1}$, в случае, когда $\lambda$ много больше толщины как верхнего, так и среднего слоев, режим „внутренних волн“ не наблюдается: амплитуды волн на всех границах раздела сравнимы с амплитудой изначально возбуждаемой волны. Когда толщина верхнего слоя сравнима с длиной волны, а толщина среднего слоя много ее меньше $\left(\lambda \sim h_{1} \gg h_{2}\right)$, наблюдается аналог режима „внутренних волн“, так как амплитуды волн на внутренних границах раздела (одна из которых изначально возбуждена) существенно превышают амплитуду волны на свободной поверхности. В случае, если длина волны сравнима с толщиной как верхнего, так и среднего слоев $\left(\lambda \sim h_{1} \sim h_{2}\right)$, начальное возбуждение средней границы раздела оказывает малое влияние на колебания верхней и нижней границ, амплитуды волн на которых существенно меньше.

Выше мы рассмотрели закономерности изменения отношений амплитуд волн, возникающих в математической модели трехслойной несжимаемой жидкости со свободной поверхностью. Говоря об устойчивой стратификации жидкости по плотности, мы не имели в виду реально протекающие в океанах и морях процессы, а только обосновывали осуществимость вышеуказанной математической модели, возможность реализации подобной структуры в естественных условиях.

\section{Заключение}

Для математической модели волн в устойчиво стратифицированной трехслойной жидкости со свободной поверхностью дисперсионное уравнение является бикубическим относительно частоты. Каждому из трех решений для квадрата частоты соответствует свой режим колебаний: либо режим движения „сплошной среды“, либо режим „внутренних волн“. В первом случае амплитуды волн на всех трех границах раздела сред имеют сравнимую величину. В режиме „внутренних волн“ значения амплитуд волновых возмущений на внутренних границах раздела сред значительно превышают амплитуду возмущения свободной поверхности. Возможность реализации этого режима существенно зависит от отношения плотностей контактирующих сред: он тем ярче проявляется, чем меньше разность плотностей сред. В трехслойной жидкости со свободной поверхностью режим „внутренних волн“ может наблюдаться при начальном возмущении как средней, так и нижней границы. Большое значение имеет и соотношение между длиной распространяющейся волны и толщиной слоев.

Начальное волновое возмущение какой-либо из границ раздела заметно сказывается на колебаниях двух других границ в случае, когда длина волны сравнима с толщиной слоев. Общий вид поверхностей раздела сред определяется одновременно существующими волнами, порожденными всеми поверхностями раздела.

Работа выполнена при поддержке гранта РФФИ № 14-01-00170-a.

\section{Список литературы}

[1] Brooke Benjamin T. // J. Fluid Mech. 1967. Vol. 29. Part 3. P. 559-592.

[2] Сретенский Л.Н. Теория волновых движений жидкости. М.: Наука, 1977. 815 с

[3] Wooyoung C., Camassa R. // J. Fluid Mech. 1999. Vol. 396. P. 1-36.

[4] Troy C.D., Koseff J.R. // J. Fluid Mech. 2005. Vol. 43. P. $107-136$.

[5] Гурьев Ю.В., Ткаченко И.В. // Изв. РАН. МЖГ. 2010. № 1. C. $22-31$.

[6] Григорьев А.И., Федоров М.С., Ширяева С.О. // Изв. РАН. МЖГ. 2010. № 5. С.130-140.

[7] Prabir D. // Phys. Fluids. 2008. Vol. 20. P. 112101.01112101.11.

[8] Kyung Sung Kim, Moo Hyun Kim // Int. J. Offshore Polar Eng. 2014. Vol. 24. N 2. P. $122-128$.

[9] Ширяева С.О., Григорьев А.И., Яковлева Л.С. // ЖТФ. 2015. Т. 85. Вып. 12. С. 40-44.

[10] Ширяева С.О., Петрушов Н.А., Григорьев А.И., Федоров М.С. // ЖТФ. 2014. Т. 84. Вып. 9. С. 31-38.

[11] Mercier M.J., Vasseur R., Dauxois T. // Nonlinear Proc. Geophys. 2011. Vol. 18. P. 193-208.

[12] Сретенский Л.Н. // Журн. геофизики. 1934. Т. 4. Вып. 3. C. $332-367$. 
[13] Корпусов М.О. // Изв. РАН. Сер. математическая. 2011. T. 75. № 4. C. 29-48.

[14] Куркин А.А. // Экологические системы и приборы. 2013. № 10. C. 43-49.

[15] Григорьев А.И., Федоров М.С., Ширяева С.О. // Изв. РАН. МЖГ. 2014. № 6. С. 37-44.

[16] Черкесов Л.В. Введение в гидродинамику и теорию волн. СПб.: Гидрометеоиздат, 1992. 264 с. 